\title{
Effects of Active Noise Control on Physiological Functions
}

\author{
Soomin LeE ${ }^{1)}$, Tetsuo KatsuUra ${ }^{2)}$, Yoshihiro Shimomura ${ }^{2)}$, Xin Xin Liu ${ }^{2)}$, Fumiyasu Konno ${ }^{3)}$, \\ Masahide ONISHI ${ }^{3)}$, Masaki TADA ${ }^{3)}$ and Kazuhisa KoTEGAWA ${ }^{3)}$ \\ 1) Graduate School of Science and Technology, Chiba University \\ ${ }^{2)}$ Graduate School of Engineering, Chiba University \\ 3) Panasonic Electronic Devices Co., Ltd \\ E-mail: yisoomin@graduate.chiba-u.jp
}

(received on September 5, 2008, accepted on August 18, 2009)

\begin{abstract}
The noise inside an automobile exerts various influences on the driver. In this context, the concept of active noise control (ANC), which reduces the noise in the automobile, has been reported. Subjective evaluation has furnished empirical evidence regarding the effects of ANC. However, few studies have investigated the effects of ANC using a physiological evaluation. Therefore, we studied the effects of ANC on the physiological functions using recorded driving noise with ANC ON and OFF conditions. Ten male subjects participated in this study. They completed four consecutive sessions: a 20 min quiet period as baseline, a 10 min noise exposure with ANC ON or OFF, a 60 min mental task period during noise exposure with ANC ON or OFF, and a $20 \mathrm{~min}$ recovery period. We measured electrocardiogram, photoplethysmogram, electroencephalogram, blood pressure (BP), baroreflex sensitivity and internal secretion indexes such as cortisol. Before and after the task, the subjects were evaluated on the Kansei-gakuin Sleepiness Scale, and after the task, they answered the NASA-TLX. Two experiments with ANC ON and OFF conditions were conducted at the same time of day on separate days. Data of the ANC ON condition and ANC OFF condition were compared by the paired $t$-test. We found that systolic $\mathrm{BP}$, diastolic $\mathrm{BP}$ and sympathetic nerve activity during the ANC ON condition were significantly lower than those during the ANC OFF condition. Furthermore, parasympathetic nerve activity during the ANC ON condition was significantly higher than that during the ANC OFF condition. The results suggested that ANC effectively reduces the stress level in physiological functions, especially the cardiovascular system.
\end{abstract}

Key words: active noise control (ANC), mental stress, physiological responses, subjective evaluation.

\section{Introduction}

The demand to prevent car accidents continues to increase, and there have been many studies on reducing the driver's burden. It has been reported that noise inside the automobile exerts various influences on the driver. Therefore, installing active noise control (ANC) technology that decreases the noise in the automobile would be an effective way to reduce the driver's burden. It is very important to confirm whether ANC influences the driver's physiological and psychological responses.

ANC has been an area of very active research for many years since the first appearance of the seminal ideas of Lueg. Today, the underlying principles are well established (Kuo et al., 1995; Nelson et al., 1992), but there are still many practical issues to be resolved. This technology was studied actively during the early 1990s, and now it is in practical use in various situations including automotive use. A wide variety of applications, including aircraft engines, vehicle interiors, and air conditioning systems as well as household appliances, have been produced (Ayala et al., 2005; Dozio et al., 2007). The concept of ANC is to reduce undesirable stationary noise by reproducing the reverse phase sound from the speaker. In other words, ANC is the only effective method of attenuating low-frequency noise. Fig. 1 shows the concept of ANC; when a reverse phase sound is added to a noise signal, the residual noise becomes smaller.

There have been many studies on the influence of ANC from the psychological point of view. According to Watanabe's study (2007), the reaction time of the performance task was shorter in the ANC ON condition. In addition, it was found that feelings of 


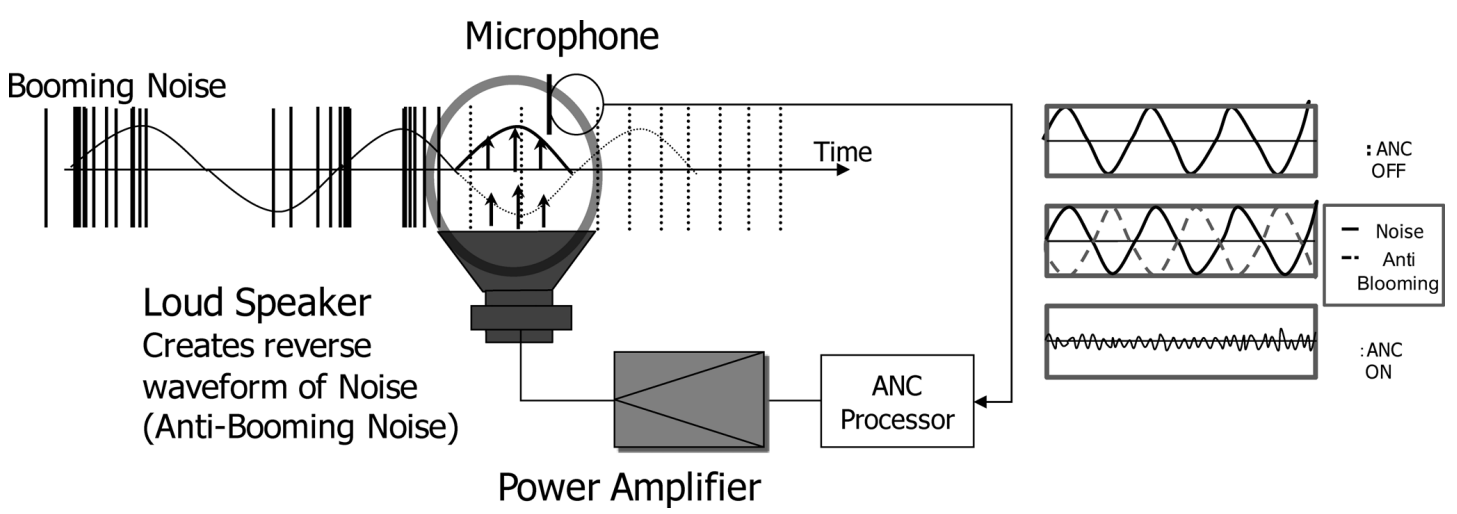

Fig. 1. The algorithms and concept of active noise control (ANC).

annoyance and depression were decreased in the ANC ON condition (Abe et al., 1997; Honda et al., 2001). Unfortunately, physiological data on the ANC effect were limited. Although Watanabe (2007) studied the effects of ANC on physiological responses such as blood pressure (BP), heart rate (HR), and electroencephalogram (EEG), they failed to find the effects of ANC on them.

On the other hand, it is well-known that noise exposure influences physiological functions. Based on early studies, it was found that BP became elevated after acute exposure to noise (Tomei et al., 2000; Liu et al., 2006), and prolonged periods of noise exposure might cause an increase in BP, thus increasing the risk of cardiovascular disease (Norvell et al., 1989). Furthermore, another independent line of research showed deleterious effects of noise exposure on the endocrine stress system. Salivary cortisol received particular attention, and was considered to be an excellent stress marker (Kirschbaum et al., 1994). Cortisol has recently been suggested to be useful tool to measure noise-related stress (Melamed et al., 1996; Bigert et al., 2005). For instance, workers who were exposed to high ambient noise levels had lower cortisol secretion, fatigue, and irritability when wearing earmuffs to reduce ambient noise levels (Doyle et al., 1996).

The purpose of this study was to clarify the influence of ANC on the human body. Thus, we studied the effects of ANC on the physiological functions and endocrine stress system by using recorded driving noise in the ANC ON and OFF conditions.

\section{Methods}

\subsection{Subjects}

Ten male subjects (21-32 years old) participated in this study. Their physical characteristics are shown in Table 1. They were asked to refrain from exercise, drinking caffeinated beverages, and smoking cigarette during the $2 \mathrm{~h}$ period immediately preceding the
Table 1. Physical characteristics of the subjects.

\begin{tabular}{cccc}
\hline Subjects & Age (yr) & Height (cm) & Weight (kg) \\
\hline A & 24 & 170 & 55 \\
B & 23 & 176 & 68 \\
E & 21 & 172 & 60 \\
F & 32 & 174 & 67 \\
G & 21 & 164 & 58 \\
H & 25 & 172 & 56 \\
I & 27 & 175 & 70 \\
J & 23 & 176 & 61 \\
\hline Mean \pm SD & $24 \pm 3.4$ & $171 \pm 4.42$ & $60 \pm 6.8$ \\
\hline
\end{tabular}

experiment. The subjects performed an auditory test before the experiment. Their hearing ability was confirmed to be normal. All subjects gave fully informed consent to participate in this study.

\subsection{Task and Noise Exposure}

The stresses consisted of arrow task and noise exposure in the ANC ON and OFF conditions. For the arrow task, an arrow which showed different directions every three seconds was displayed on the screen, and the subjects were asked to push the appropriate button according to the direction of the arrow. The noise was obtained in a vehicle (V-6 engine, $3.5 l$ ) that ran at $48 \mathrm{~km} / \mathrm{h}$ on a driving test course in the United States. The noise was recorded for $30 \mathrm{~s}$, and we assembled these short pieces to make a $60 \mathrm{~min}$ recording. We used this $60 \mathrm{~min}$ noise for a $60 \mathrm{~min}$ trial (ANC OFF condition). The noise was modulated using the feedforward method in the ANC technology (ANC ON condition) (Reddy et al., 2008; Yuan., 2008). The Leq (equivalent sound level) of ANC ON and OFF conditions measured in an auditory laboratory were $65.6 \mathrm{dBA}$ and $70.7 \mathrm{dBA}$, respectively. The noise stimuli with ANC ON and OFF were reproduced by a high-fidelity loudspeaker system (speaker: REY-AUDIO, RM-7V; power amplifier: JDF, HQS3200UPM). 


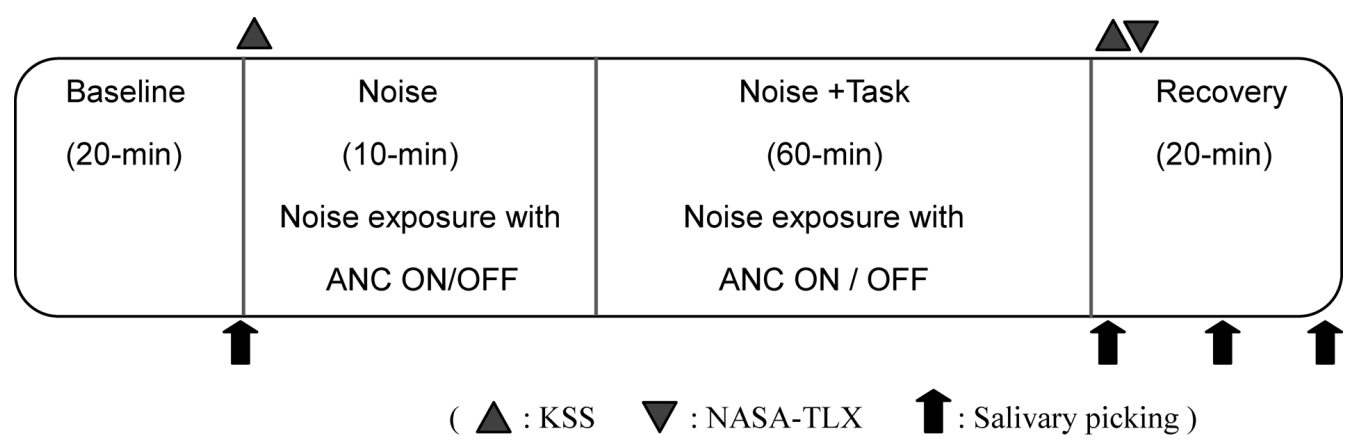

Fig. 2. The experiment protocol.

Noise: the subjects were exposed to the noise while they relaxing.

Noise + task: the subjects were exposed to the noise while they conducted arrow task as a mental task.

\subsection{Procedure}

Two experiments were conducted at the same time of day on separate days and under the same conditions with the exception of the ANC condition (ANC $\mathrm{ON}$ or OFF). Subjects were asked to relax for at least 15 min after they arrived at the auditory laboratory. They completed four consecutive sessions: $20 \mathrm{~min}$ rest on a chair in a quiet condition $(22 \mathrm{dBA})$ as the baseline, $10 \mathrm{~min}$ noise exposure with ANC ON or $\mathrm{OFF}$ as the noise period, a $60 \mathrm{~min}$ arrow task period during noise exposure with ANC ON or OFF as the noise and task period, and a 20 min quiet recovery period. The noise is similar to $10 \mathrm{~min}$ noise period and $60 \mathrm{~min}$ arrow task period. We intended to confirm the effect of the noise only while the subjects took a rest during the noise period. We also measured to confirm the effect of the noise when the subjects conducted the arrow task during the noise and task period. Subjects were told to rest and physically relax throughout the experiment. The total noise exposure time was 70 min (Fig. 2). The order of the two ANC conditions was counterbalanced between the subjects.

\subsection{Measurement Items}

According to previous studies (Tomei et al., 2000; Melamed et al., 1996; Liu et al., 2006), noise influences the cardiovascular system and the endocrine system. Therefore, we measured BP, baroreflex sensitivity (BRS), and HR as the cardiovascular indexes and salivary cortisol as the endocrine index to confirm whether ANC influenced the human body. Furthermore, we monitored the central nervous system and made a subjective evaluation to confirm whether ANC had an influence on drowsiness. We measured the following items.

\section{Physiological indexes}

EEG activity was recorded with $\mathrm{Ag} / \mathrm{AgCl}$ electrodes affixed with electrode paste on the $\mathrm{Pz}$ and $\mathrm{Fz}$ electrode sites of the international 10-20 system.
Linked earlobes were used as a reference with a forehead ground. A bipolar electrooculogram (EOG) was recorded with electrodes placed above and below the left eye. The EEG and EOG were amplified by appropriate devices (BIOPAC Systems, EEG100B and EOG100B, respectively). The high-pass filter for the EEG was set at $1.0 \mathrm{~Hz}$. EEGs were fast Fourier transformed for each $5.12 \mathrm{~s}$ of data not including artifacts such as ocular movement. We then obtained the relative power density of the alpha wave $(8-13 \mathrm{~Hz} / 4-30$ Hz) (Yoto et al., 2007).

Continuous measurements of beat-to-beat BP were obtained with an ambulatory noninvasive BP monitor (TNO, Portapres). Beat-to-beat systolic BP and systolic-to-systolic pulse intervals were analyzed for BRS using the method of sequential analysis (Parati et al., 1989; Hughson et al., 1993; Gizdulich et al., 1996).

An electrocardiogram (ECG) was recorded using an amplifier (BIOPAC System, ECG 100B), and digitized to derive heart rate variability (HRV) and HR using signal processing software (PTC, Mathcad). The high frequency (HF) components and low frequency (LF) components were integrated $0.05-0.15$ $\mathrm{Hz}$ and $0.15-0.40 \mathrm{~Hz}$ of the power spectra, respectively (Pomeranz et al., 1985; Pagani et al.,1986). Sympathetic nerve activity (LF/HF) and parasympathetic nerve activity $(\mathrm{HF} /(\mathrm{LF}+\mathrm{HF}))$ were calculated.

Photoplethysmogram (PTG) was recorded using an amplifier (BIOPAC System, PPG 100B), a low-pass filter was set at $3.0 \mathrm{~Hz}$, and a high-pass filter was set at $0.5 \mathrm{~Hz}$. We measured the $\mathrm{b} / \mathrm{a}$ ratio of second derivative of PTG (SDPTG), which was considered to reflect the distensibility of the vascular wall (Takazawa et al., 1993 and 1998; Otsuka et al., 2006).

All signals of the physiological indexes were converted from analog to digital at a $1 \mathrm{kHz}$ sampling rate (A/D converter: BIOPAC Systems, MP100), and were stored in a computer. 


\section{Cortisol measurement}

The saliva samples were taken using the salivette saliva sampling device. The subjects were asked to chew on a sterile dental swab for exactly $3 \mathrm{~min}$. The saliva samples were subsequently stored at $-20^{\circ} \mathrm{C}$ in the laboratory until being thawed for assay. The amount of cortisol secretion was determined with the salivary cortisol ELISA kit. To assess noise exposure-induced stress and to evaluate the difference in stress between the two noise conditions, four saliva samples were collected at different times during the experiment. Exact details of the saliva sampling times are given in Fig. 2.

\section{Subjective evaluations}

NASA-TLX is one of the typical subjective evaluation methods that measure mental stress, and this test is used widely. It consists of six standards, that is, an intellectual, perceived demand, a body demand, the time pressure, the work result, the effort, and the frustration. NASA-TLX was measured after the task.

KSS (Kwansei-gakuin Sleepiness Scale) test is used to evaluate drowsiness. This value calculates the score based on 22 items from which the rating scale value is derived as an average. KSS was measured before the noise period and after the task period.

\subsection{Statistical Analysis}

We calculated the mean values of every $5 \mathrm{~min}$ for all indices. We normalized the values by converting them into mean 0, standard deviation 1. Data for comparing the ANC ON condition with the ANC OFF condition were tested using the paired $t$-test. The level of statistical significance was set at 0.05 .

\section{Results}

\subsection{Physiological Responses}

Figure 3 and Fig. 4 show the results of the systolic $\mathrm{BP}$ and the diastolic BP in the ANC ON and ANC OFF conditions. Systolic BP during the ANC ON condition in the noise and task period was significantly $(p<0.01)$ lower than that during the ANC OFF condition. Diastolic BP in the noise and task period during the ANC ON condition was also significantly $(p<0.05)$ lower than that during the ANC OFF condition. On the other hand, no significant difference was observed in BRS between ANC ON and ANC OFF conditions. Figure 5 shows sympathetic nerve activity (LF/HF) in the ANC ON and ANC OFF condition. The sympathetic nerve activity during ANC ON condition in the noise periods tended to be lower than that during the ANC OFF condition $(p=0.07)$. Figure 6 shows the results of the parasympathetic nerve activity in the ANC ON and ANC OFF conditions. Parasympathetic nerve activity during the ANC ON

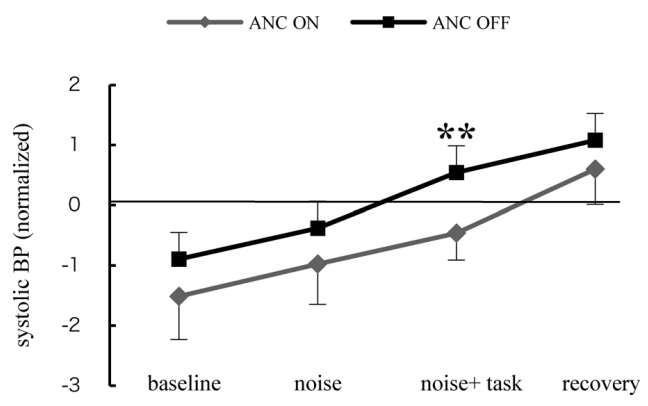

Fig. 3. Systolic BP in the ANC ON/OFF conditions $(* *: p<0.01$, mean $\pm \mathrm{SE})$.

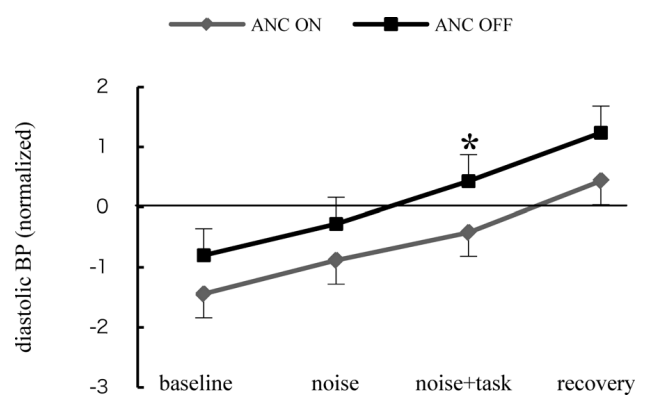

Fig. 4. Diastolic BP in the ANC ON/OFF conditions $(*: p<0.05$, mean $\pm \mathrm{SE})$.

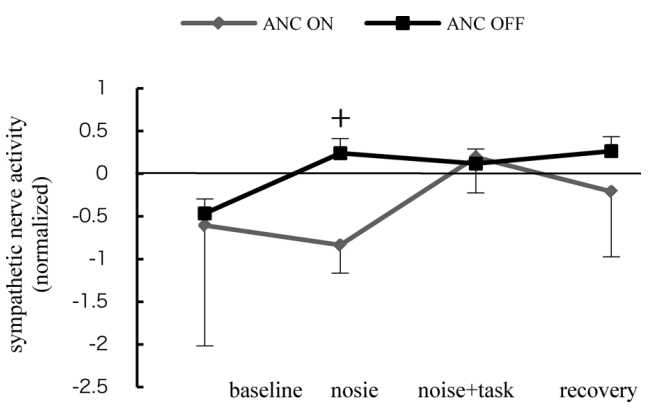

Fig. 5. Sympathetic nerve activity in the ANC ON/ OFF conditions $(+: p<0.1$, mean $\pm \mathrm{SE})$.

condition was significantly $(p<0.05)$ higher at the noise periods. In contrast, the relative power density of $\mathrm{Pz}$ and $\mathrm{Fz}, \mathrm{HR}$ and amplitude ratio (b/a) of SDPTG were not significantly different between the ANC ON and ANC OFF conditions.

\subsection{Cortisol Secretion and Subjective Evaluation}

The cortisol secretion showed no significant difference between the ANC ON and ANC OFF conditions (Fig. 7). Figure 8 shows the results of KSS in the ANC ON and ANC OFF conditions. KSS during the ANC ON condition tended to be higher than that during the ANC OFF condition $(p=0.06)$. On the other hand, NASA-TLX, the reaction time and correct rate of the task showed no significant differences between the ANC ON and ANC OFF conditions. 


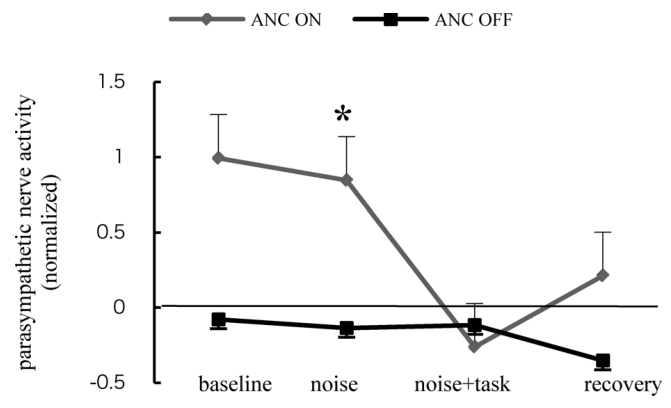

Fig. 6. Parasympathetic nerve activity in the ANC ON/OFF conditions $(*: p<0.05$, mean \pm SE).

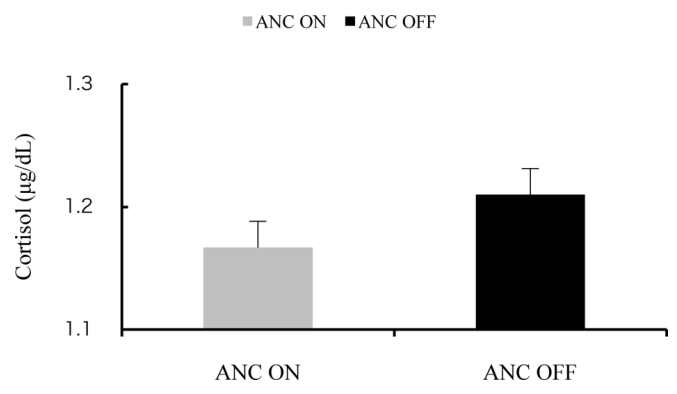

Fig. 7. Cortisol secretion in the ANC ON/OFF Conditions (mean $+\mathrm{SE})$.

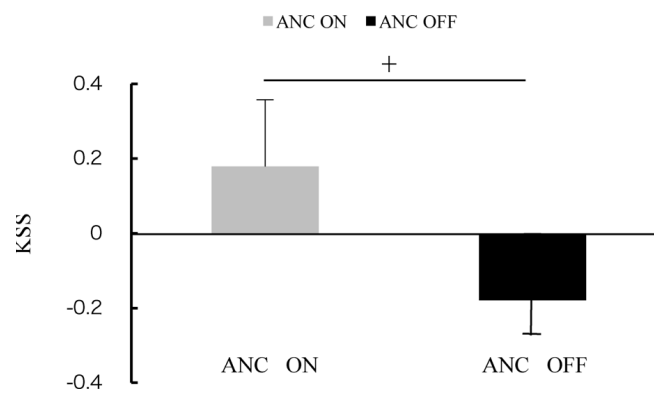

Fig. 8. KSS in the ANC ON/OFF conditions $(+: p<0.1$, mean $+\mathrm{SE})$.

\section{Discussion}

The present study compared the physiological responses and subjective evaluations in the ANC ON condition and the ANC OFF condition. We found that systolic BP and diastolic BP during the ANC ON condition were significantly lower than those during the ANC OFF condition in the noise and task period. Parasympathetic nerve activity during the ANC ON condition was significantly higher than that during the ANC OFF condition in the noise periods. Furthermore, sympathetic nerve activity during the ANC $\mathrm{ON}$ condition in the noise periods tended to be lower than that during the ANC OFF condition. According to these results, it is clarified that the burden of cardiovascular system was reduced by ANC. This is the first research to find that the relationship between the ANC effect and the burden can be verified by measuring cardiovascular parameters.
In contrast, the relative power density of $\mathrm{Fz}$ and $\mathrm{Pz}$ was not significantly different between the two conditions. It is suggested that ANC effectively reduces the burden not in the central nervous system but in the cardiovascular system.

On the other hand, we could not determine the effect of ANC on cortisol secretion. The result for cortisol secretion agreed with that in the previous study (Watanabe et al., 2007). The cortisol secretion may be related to the kind of stress exposure time and intensity of the stress. Nakane (1999) reported that the cortisol reached its maximum level right after a speech task, and intense exercise. In addition, previous studies of humans and animals found an increase of cortisol by the noise exposure of longer duration, higher noise levels, or both (Melamed et al., 1996; Herbert et al., 2009).

However, the Leq of ANC ON and ANC OFF conditions (65.6 dBA and $70.7 \mathrm{dBA})$ in the present study were lower than those of previous study ( $85 \mathrm{dBA}$ and $95 \mathrm{dBA})$ (Melamed et al., 1996). Furthermore the noise exposure time was shorter than in the previous studies (Melamed et al., 1996; Herbert et al., 2009). The stress caused by the noise exposure in the present study may not have been intensive. In other words, cortisol reacts to intensive stress, but does not react to continuous and relatively low stress. It is estimated that the stress of the noise which we used is weaker than that in the previous study (Nakane, 1999). Thus, the difference of the hormone levels of the two ANC conditions might not be appeared.

Meanwhile, KSS during the ANC ON condition tended to be higher than that during the OFF condition throughout the sessions $(p=0.06)$. It was shown that the effect of ANC on the KSS was coincident with the responses in the cardiovascular system. We thought that the subjects experienced relaxation as a results of the ANC which reduces the burden in the human body. In terms of results it is expected in future that the ANC technology will help to improve the interior sound environment of the vehicle.

\section{Conclusion}

We verified that $\mathrm{ANC}$ reduces the burden in the human body, especially the cardiovascular system, by reducing undesirable driving noise. Furthermore, we confirmed the effect of ANC by measuring the physiological responses. Thus, we found that BP, sympathetic nerve activity, and parasympathetic nerve activity were usable indexes to evaluate physiological responses to ANC.

\section{References}

Ayala Botto M., Sousa J. M. C. (2005) Intelligent active noise control applied to a laboratory railway coach 
model. Control Engineering Practice 13(4): 473-484.

Abe S., Watanabe Y., Hamada H. (1997) Subjective evaluation of the effect for active noise control. INTERNOISE 1: 533-536.

Bigert C., Bluhm G., Theorell T. (2005) Saliva cortisol a new approach in noise research to study stress effects. Int J Hyg Environ Health 208(3): 227-230.

Doyle A., Hucklebridge E., Evans P., Clow A. (1996) Salivary monoamine oxidase $\mathrm{A}$ and $\mathrm{B}$ inhibitory activities correlate with stress. Life Sci 59: 1357-1362.

Dozio, L., Mantedgazza, P. (2007) General-purpose processors for active vibro-acoustic control: Discussion and experience. Control Engineering Practice 15(2): 163-176.

Gizdulich P., Imholz BP., van den Meiracker A.H., Parati G., and Wesseling K.H. (1996) Finapres tracking of systolic pressure and baroreflex sensitivity improved by wave form filtering. J Hypertens 14: 243-250.

Hebert S., Lupien S. (2009) Salivary cortisol levels, subjective stress, and tinnitus intensity in tinnitus suffers during noise exposure in the laboratory. Int. J Hygiene and environmental Health 212: 37-44.

Honda S., Watanabe Y., Hamada H. (2001) Subjective investigation for active noise control system. The Japan Society of Mechanical Engineers: 59-62.

Hughson R.L., Quintin L., Annat G., Yamamoto Y., Gharib C. (1993) Spontaneous baroreflex by sequence and power spectral methods in humans. Clin Physiol. 13: 663-676.

Kirschbaum C., Hellhammer D. H. (1994) Salivary cortisol in psychoendocrine research: recent developments andapplications. Psychoneuroendocrinology 19: 313-333.

Kuo S., Morgan D. (1995) Active noise control systems: Algorithms and DSP implementations. New York: Wiley.

Liu X., Iwanaga K., Shimomura Y., Katsuura T. (2007) Comparison of stress responses between mental tasks and white noise exposure, J Physio Anthropol 26: 165-171.

Melamed S., Bruhis S. (1996) The effects of chronic industrial noise exposure on urinary cortisol, fatigue and irritability-A controlled field experiment. J Occup Environ Med. 38: 252-256.

Nakane H. (1999) Salivary Chromogranin A as index of Psychosomatic stress response. Technical Journal R\&D Review of Toyota CRDL 34 (3): 17-22.

Nelson P., Elliot S. (1992) Active control of sound. New York: Academic Press.

Norvell N. Roth. D. L. (1989) Cardiovascular reactivity and silent Ischemia in response to mental stress in symptomatic and asymptomatic coronary artery disease patients: results of a pilot study. Ciln Cardiol 12: 634-638.

Ostuka T., Kawada T., Katsumata M. (2006) Utility of second derivative of the finger Photoplethysmogram for the Estimation of the risk of Coronary Heart Disease in the general population. Circ J 70: 304-310.

Pagani M., Lombardi F., Guzzetti S., Rimoldi O., Furlan R., Pizzinelli P., Sandrone G., Cerutti S., Malliani A. (1986) Power spectral analysis of heart rate and arterial pressure variabilities as a marker of sympatho-vagal interaction in man and conscious dog. Circ Res 59: 178-193.

Parati G., Casadei R., Groppelli A., Di Rienzo M., Mancia G. (1989) Comparision of finger and intra-arterial blood pressure monitoring at rest and during laboratory testing. Hypertension 13: 647-655.

Pomeranz B., Macaulay RJB., Caudill MA., Kutz I., Adam D., Gordon D., Kilborn KM., Barger AC., Shannon DC., Cohen RJ., Benson H.(1985) Assessment of autonomic function in humans by heart rate spectral analysis. Am J Physiol 248: H151-H153.

Reddy RM., Panahi IM. (2008) Comparision between hybrid feedforward-feedback, feedforward, and feedback structures for active noise control of fMRI noise. Conf Proc IEEE Eng Med Biol Soc: 266-269.

Takazawa K., Fujita M., Yabe K. (1993) Clinical usefulness of the second derivative of a plethysmogram (acceleration plethysmogram). J Cardiol 23: 207-217.

Takazawa K., Tanaka N., Fujita M. (1998) Assessment of vasoactive agents and vascular aging by the second derivative of photoplethysmogram waveform. Hypertension 32: 365-370.

Tomei F., Fantini S., Tomao E. (2000) Hypertension and chronic exposure to noise. Arch Environ Health 55: 319-325.

Watanabe Y., Utsumi S., Murai E., Hamada H., Mitsuhata S., Abe S. (2007) Subjective effective of ANC system and it's evaluation methods. The Institute of Electronics, Information and Communication Engineers: 1-6.

Yoto A., Katsuura T., Iwanaga K., Shimomura Y. (2007) Effects of object color stimuli on human brain activities in perception and attention referred to EEG alpha band response. J Physiol Anthropol 26: 373-379.

Yuan. J (2008) Model independent control of lightly damped noise/vibration systems. J Acoust Soc Am 124(1): 241-246. 